\title{
Influence of Morphology on Transport Properties and Interfacial Resistance in Nanoporous Carbons
}

\author{
Lang $\operatorname{Liu}^{\mathrm{a}, \mathrm{b} *}$ and Suresh K. Bhatia ${ }^{\mathrm{b}^{*}}$
}

a School of Chemical Engineering, The University of Queensland Brisbane, QLD 4072, Australia

b Key laboratory of low-grade Energy Utilization Technologies and Systems, Ministry of Education, School of Energy and Power Engineering, Chongqing University, Chongqing 400044, China

\section{Simulation details}

In Zhu et al. ${ }^{1}$ method, a collective coordinate, $n$, was introduced to quantify the net amount of fluid molecules permeating through the nano channel connecting the bulk reservoirs, with the mean square displacement of $n$ obeying the Einstein relation,

$$
\left\langle n^{2}(t)\right\rangle=2 D_{n} t
$$

where $D_{n}$ was defined as the diffusion coefficient of $n$. Further, the relation between the flow rate, $J_{n}$, driven by a chemical potential (pressure) drop through the nano channel and the collective diffusion coefficient $D_{n}$ was obtained as,

$$
J_{n}=-\frac{\Delta \mu}{R T} D_{n}
$$

With $\Delta \mu$ being very small, i.e. the chemical potentials, $\mu_{1}$ and $\mu_{2}$ at the entrance and exit of carbon structure being close to each other, so that $\mu_{1} \cong \mu_{2}$, we have

$$
J_{n} \cong \frac{\mu_{1}-\mu_{2}}{R T} D_{n}(\mu)=\mathrm{J}_{\mathrm{c}} \cong \frac{A_{c} \rho_{c}(\mu) D_{0}^{\text {finite }}(\mu)}{R T} \cdot \frac{\mu_{1}-\mu_{2}}{L_{c}}
$$


where, $J_{c}$ is the flow rate calculated based on $D_{0}^{\text {finite }}$ that is the corrected diffusivity of the adsorbate in the finite carbon structure in the presence of interfacial resistance and $\mu$ is the mean chemical potential of the system, $\mu=\frac{1}{2}\left(\mu_{1}+\mu_{2}\right) . \rho_{c}, A_{c}$ and $L_{c}$ are the number density of the adsorbate $\left(\mathrm{mol} / \mathrm{nm}^{3}\right)$ in the solid structure, the cross sectional area of the solid structure that is perpendicular to the flow direction and the thickness of the carbon structure. We note $A_{c}$ for the CNT is the cross-sectional area of the CNT, not the flange area. Therefore, $D_{0}^{\text {finite }}$ can be extracted from $D_{n}$ as

$$
D_{0}^{\text {finite }}(\mu)=\frac{D_{n}(\mu) L_{c}}{\rho_{c}(\mu) A_{c}}
$$

\section{Results}

\subsection{Effect of morphology on the internal diffusion}
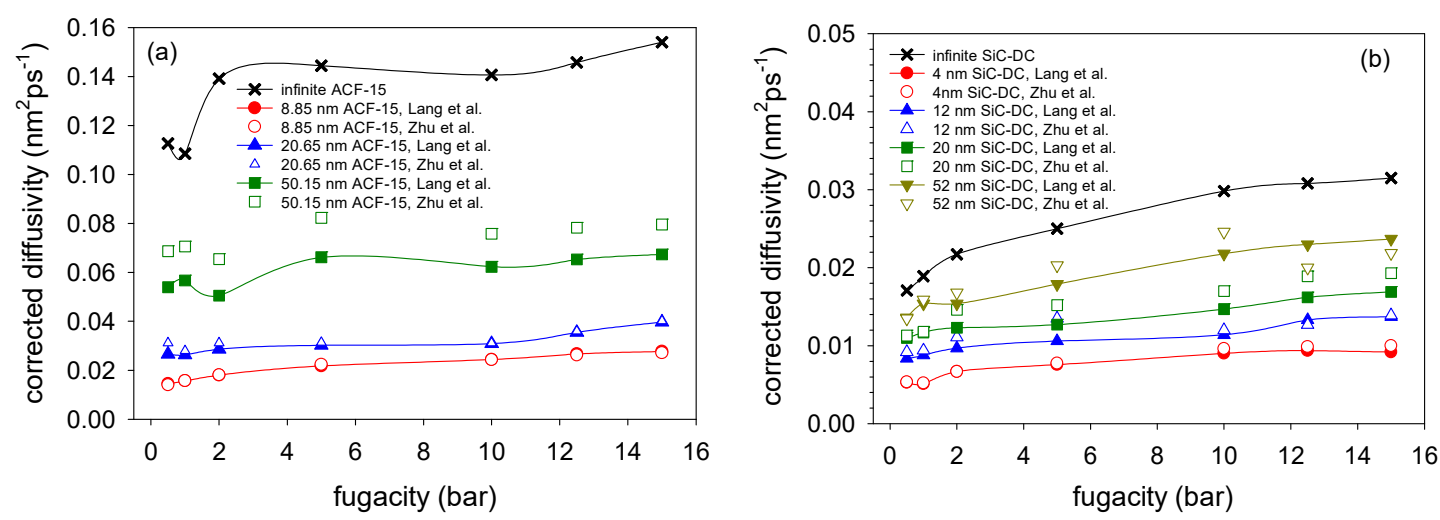

Figure S1. Variation of the corrected diffusivities of methane in the $X$ direction with pressure in (a) ACF-15, and (b) SiC-DC, calculated from our method ${ }^{2}$ and Zhu et al. method ${ }^{1}$.

It can be seen from Figures S1 (a) and (b), the corrected diffusivities calculated from our method ${ }^{2}$ and the method proposed by Zhu et al. ${ }^{1}$ match each other very well in the finite ACF-15 and SiCDC. During our simulations, we find that sufficiently long simulation runs are important to obtain the strictly linear trends of the COM-MSD, and the mean square displacements of the collective 
number with time in the system with enhanced thickness. Therefore, it is anticipated that the small discrepancy between the results calculated from these two methods in the $50.15 \mathrm{~nm}$ ACF15 can be considerably reduced by extending the simulation time or by increasing the number of independent equilibrium molecular dynamic runs.

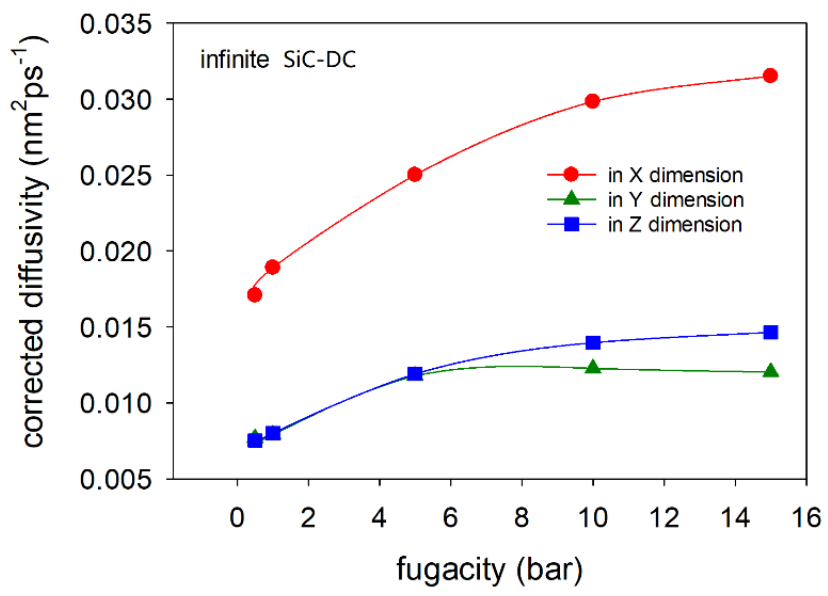

Figure S2. Variation of the corrected diffusivities of methane in different directions with pressure in the infinite SiC-DC, calculated from EMD simulations, at $300 \mathrm{~K}$.

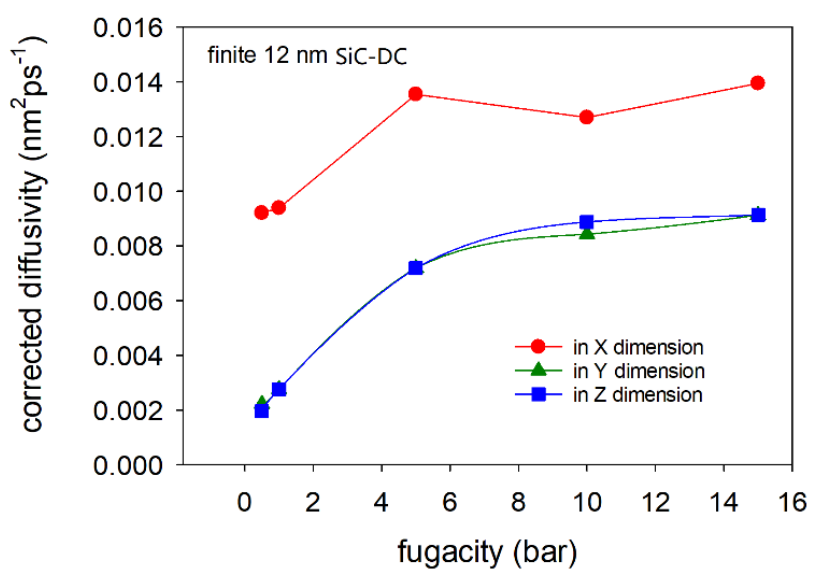

Figure S3. Variation of the corrected diffusivities of methane in different directions with pressure in the $12 \mathrm{~nm}$ thick SiC-DC, calculated from EMD simulations at $300 \mathrm{~K}$. 


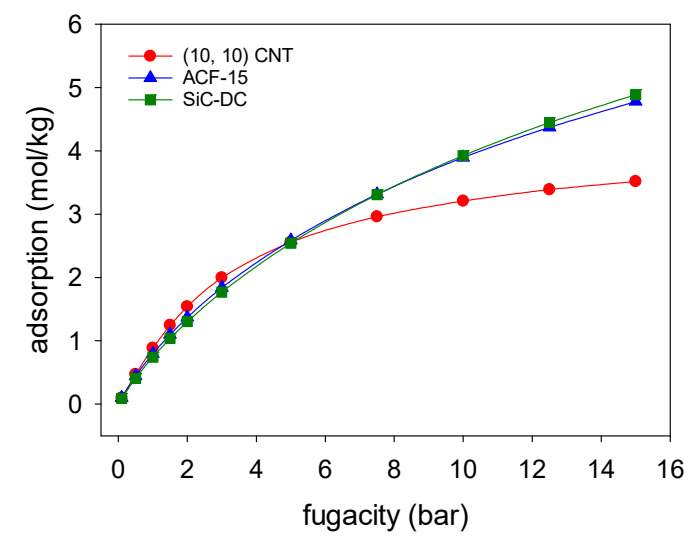

Figure S4. Adsorption isotherms of methane in the infinite $(10,10)$ CNT, ACF-15 and SiC-DC, at $300 \mathrm{~K}$.

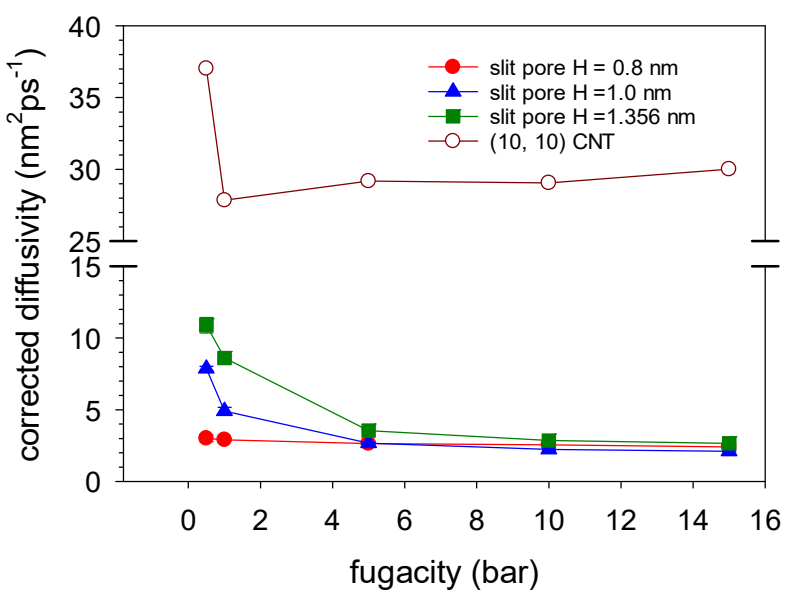

Figure S5. Variation of the corrected diffusivities of methane in graphene slit pores calculated from EMD simulations, at $300 \mathrm{~K}$.

The pore widths of the graphene slit pores are determined as the center to center distance between two carbon atoms in the opposite walls. The diffusivity of $\mathrm{CH}_{4}$ in the slit pore was calculated using the NEMD method presented in detail in our previous work ${ }^{2}$, with the periodic boundary conditions being applied in the $X$ and $Y$ directions, which are perpendicular to the flow direction, in the $Z$ direction. 


\subsection{Effect of morphology on diffusion in the presence of interfacial resistance}
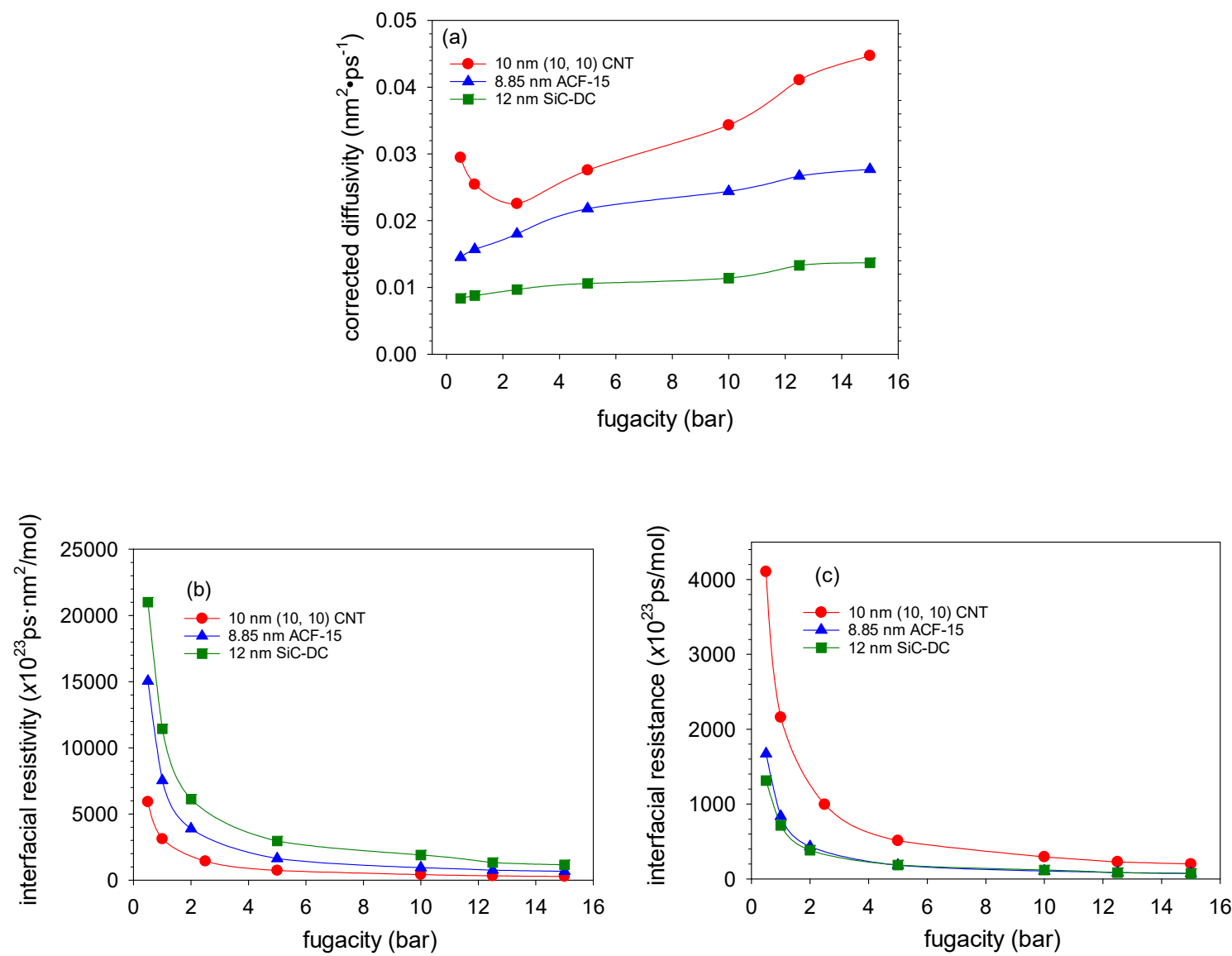

Figure S6. Variation of (a) the corrected diffusivity of methane, (b) interfacial resistivity, and (c) the interfacial resistance, with fugacity in the finite $(10,10)$ CNT, ACF-15 and SiC-DC.

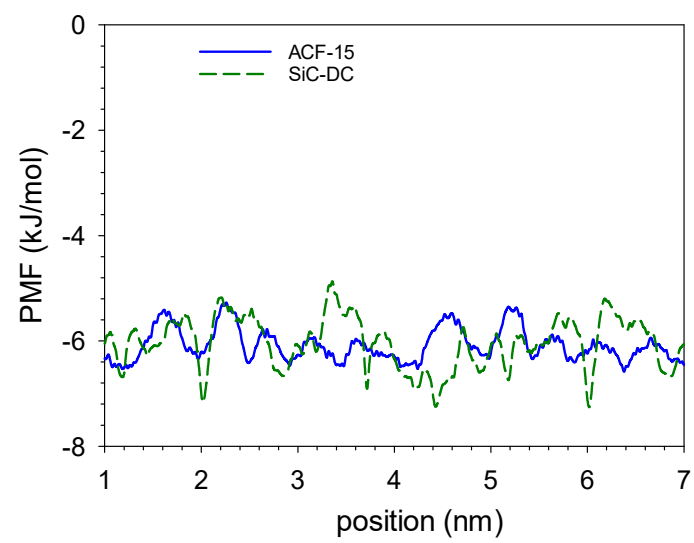

Figure S7. PMF profiles in the ACF-15 and SiC-DC structures, at 5 bar and $300 \mathrm{~K}$. Position 1 $\mathrm{nm}$ was a randomly chosen position in the carbon structure. 
Note that the thicknesses of the unit cells of ACF-15 and SiC-DC in the $X$ direction are $2.95 \mathrm{~nm}$ and $4 \mathrm{~nm}$, and we have plotted the potential of mean force (PMF) profiles over $6 \mathrm{~nm}$ continuous region in Figure S7, which means that the PMF profiles have covered more than a single unit cell of the ACF-15 and SiC-DC.
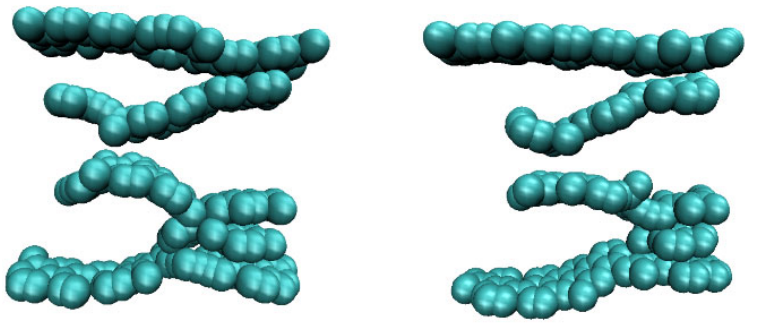

(a) ACF-15 entrance slice

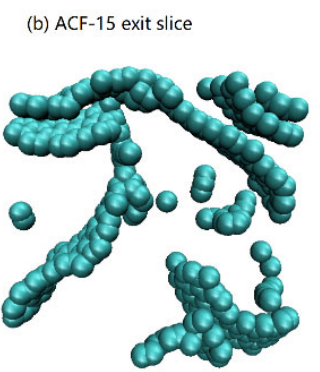

(d) SiC-DC exit slice

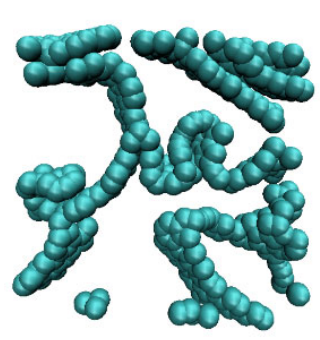

(c) SiC-DC entrance slice

Figure S8. Atomic structures of the entrance slices of (a) ACF-15, and (c) SiC-DC and exit slices of (b) ACF-15, and (d) SiC-DC.

\section{References}

(1) Zhu, F.; Tajkhorshid, E.; Schulten, K., Collective Diffusion Model for Water Permeation through Microscopic Channels. Physical Review Letters 93, 2004, 224501-1-224501-4.

(2) Liu, L.; Hu, C.; Nicholson, D.; Bhatia, S. K., Inhibitory Effect of Adsorbed Water on the Transport of Methane in Carbon Nanotubes. Langmuir 33, 2017, 6280-6291. 